\title{
RESOLUCIÓN DE PROBLEMAS Y ENSEÑANZA DE LAS CIENCIAS NATURALES
}

Margie N. Jessup C., Ph. D. **

\begin{abstract}
It has been wrongly cons,dered for a long time that resolving problems is a fundamental science activity. This activity is a characteristic when considering others human activities. Nevertheless, such activity constitutes an intrinsical element of human common life in ah spheres of his or her daily life and both individual and social life, it has gone into of the scientific field to be applied to anothers. This has been thought to be an expression of creative thinking.

Due to it, since education in science different alternatives and approaches have been proposed. focusing in solving of problems like a process that con tributes to the appropriation of a scientific culture, that it is put at service of human being and it contributes well-being and of course, to elevate his or her qualty of life and of the groups it involves.

Given the importance of the theme for education, in this paper it's shown the Line of Investígation about solving problems in the frame of Doctorate in Education with emphasis in Education in Natural Science. Consequently, it presents succinctly the fundamental developings in the field, in order to point out the lineaments of this line of investigation.
\end{abstract}

\section{INTRODUCCIÓN}

La búsqueda de calidad en la enseñanza - para el caso particular de las ciencias naturales - ha llevado al desarrollo de diferentes estrategias pedagógicas y de investigación en este campo. Los resultados de dichas investigaciones señalan múltiples causas de los diversos niveles de aprendizaje (memorístico, creativo, innovativo), relacionados con aspectos que van desde el conocimiento de la disciplina que se enseña hasta la aplicación de diferentes alternativas de enseñanza-aprendizaje, sin dejar de lado otros como concepciones, contextos, actitudes y habilidades, tanto de estudiantes como de profesores.

Para el caso colombiano, frente a los bajos resultados obtenidos por estudiantes colombianos en el Tercer Estudio Internacional sobre ciencias y matemáticas - TEIMC(Summa, 1997), así como a requerimientos que se presentan al sector educativo en sus diferentes niveles y modalidades, relacionados con la carencia de desarrollo de pensamiento creativo en sus estudiantes así como de preparación para asumir responsable-mente la cotidianidad y contribuir a la creación de futuro en el país, la línea de investigación aquí presentada reviste importancia por cuanto existen a nivel mundial desarrollos teóricos que desde diferentes perspectivas curriculares, resaltan la

\footnotetext{
Profesora Asociada de T.C Departamento de Biología Universidad Pedagógica Nacional. Programa Interinstitucional de Doctorado en Educación
} 
importancia del trabajo educativo encaminado hacia el desarrollo del pensamiento, particularmente en lo relacionado con procesos de resolución de problemas.

Por ejemplo, Garret (1988) referenciando a Blough (1942), Stollberg (1956) y Turner (1957), señala claramente cómo existe una antigua y ampliamente sostenida creencia de que resolver problemas es una actividad fundamental de la ciencia", que la diferencia de otras actividades humanas. Este autor plantea que por el contrario, el proceso de resolución de problemas trasciende el campo científico pues incide en otras esferas de la vida humana a niveles individual y social, siendo considerado como una expresión del pensamiento creativo. Por consiguiente, este proceso ha sido tomado en algunos enfoques como actividad central en la educación en ciencias

\section{ACERCA DE LOS PROBLEMAS Y SU RESOLUCIÓN}

En concordancia con la diversidad de cuestionamientos relacionados con la resolución de problemas, bajo esta denominación se incluye gran cantidad de tareas diferentes, hecho que de acuerdo con los planteamientos de Cohen (1977) ha contribuido a dificultar su interpretación teórica. Por ello, es conveniente presentar sucintamente qué se considera un problema en el campo de la enseñanza de las ciencias naturales, qué tratamiento ha habido por parte de diferentes autores y cómo se clasifican dichos problemas, así como también qué se considera resolución de problemas en este ámbito, lo cual permite comprender mejor el posicionamiento de la línea.

\section{¿QUÉ ES UN PROBLEMA?}

Algunos autores definen el término "problema" como una situación estimulante para la cual el individuo no tiene respuesta; en otras palabras, el problema surge cuando el individuo no puede responder inmediata y eficazmente a la situación (Woods y coautores, 1985).

Si se tienen en cuenta los planteamientos de Perales Palacios (1993), por problema puede entenderse cualquier situación prevista o espontánea que produce por un lado, un cierto grado de incertidumbre y por el otro, una conducta tendiente a la búsqueda de su solución.

Gil y colaboradores (1988) por su parte, consideran como problema una situación que presenta dificultades para las cuales no existen soluciones evidentes, pues una vez conocidas éstas, dejan de constituir problemas.

A su vez. Garret define el problema como una situación enigmática" es decir, aquella que no es ni solucionable ni resoluble sino sólo comprensible A estas situaciones el autor las denomina "problemas verdaderos", mientras aquellas que potencialmente pueden ser resueltas dentro de un paradigma, las denomina "rompecabezas" $(1984,1987)$. De igual manera este autor plantea que cada persona, en dependencia de su personalidad de las estrategias o recursos de que disponga y de su conocimiento, puede tomar una determinada situación bien como problema, bien como rompecabezas, lo cual lleva a pensar que el considerar una situación dada como problema o no, es algo estrictamente personal.

Esto concuerda con los planteamientos de numerosos autores según los cuales, si para la solución de una determinada situación se requiere sólo la aplicación de un algoritmo - 
entendido éste como una prescripción establecida y completamente determinada previamente de la forma de actuar - ésta no puede ser considerada como un problema. Si por el contrario, para su solución se hace indispensable seleccionar o integrar dos o más algoritmos mediando procesos de análisis y razonamiento, ésta podría ser considerada un problema independientemente de si tiene una o más soluciones. Lo expuesto lleva a pensar, que en múltiples ocasiones aquello que es considerado por los docentes de ciencias como problema, no pasa de ser un simple ejercicio y que en consecuencia, lo que determina si la situación planteada por el profesor constituye o no un problema, son las etapas que implica su resolución.

A partir de los planteamientos anteriores, se puede inferir la existencia de una tipificación de problemas. Efectivamente, Frazer (1982) plantea que existen dos tipos de problemas: los "artificiales" y los reales". Al primer tipo corresponden aquellos problemas cuya solución es conocida por la persona que los plantea, mientras los reales son aquellos que o no tienen solución o no se les conoce. Esto nos lleva indudablemente a la idea de que en concordancia con los desarrollos científicos actuales, un problema real en el campo de las ciencias naturales no debe ubicarse necesariamente en el marco de la física, la química o la biología, pero sí puede tener un fuerte componente de física, biología o química. Por consiguiente, resulta pertinente pensar en una resolución de problemas desde el punto de vista interdisciplinar o más aún, transdisciplinar, llevado al ámbito educativo.

Para Frazer los problemas artificiales pueden tener o no un objetivo dirigido y ser cerrados o abiertos, según posean una única solución o un número variable de ellas, en forma correspondiente. Sin embargo a diferencia de este autor, Garrett considera que los denominados por él "rompecabezas" pueden ser cerrados cuando tienen una o varias soluciones igualmente correctas y abiertos, cuando puede haber para ellos una o más respuestas que no son ni correcta(s) ni incorrecta(s) en términos absolutos, sino la(s) más adecuada(s) para el conjunto de circunstancias que rodean dicho problema.

Por último vale la pena reflexionar acerca de cuál es la diferencia esencial entre un problema experimental y otro de lápiz y papel Parece que desde el punto de vista del planteamiento, ninguna. De cualquier manera, un problema experimental lleva en si mismo el planteamiento teórico adecuado.

De igual forma cabe el interrogante. ¿Qué es resolver un problema?

\section{¿EN QUÉ CONSISTE LA RESOLUCIÓN DE PROBLEMAS?}

Diferentes autores conciben la resolución de problemas de diversas manera. Para Garret por ejemplo, resulta más afortunado referirse a "enfrentarse" a un problema que a "solucionarlo"; en ese sentido considera que el enfrentarse a un problema implica un proceso de pensamiento creativo y define la creatividad en términos de originalidad y utilidad de una posible solución a una situación dada.

Frazer por su parte, considera que la resolución de problemas constituye un proceso en el cual se utiliza el conocimiento de una determinada disciplina, así como las técnicas y habilidades de ella para salvar la brecha existente entre el problema y su solución. No obstante, debido a que en este tipo de definición no se consideran las condiciones propias del sujeto que resuelve el problema, los representantes de la psicología gestaltiana consideran a este proceso como algo productivo, donde el sujeto que resuelve un 
problema requiere un cierto periodo de "incubación" seguido de una repentina 'intuición", gracias a la cual logra reorganizar mentalmente el problema (Meyer, 1977).

Otros autores como Kempa (1986) consideran que la resolución de problemas constituye un proceso mediante el cual se elabora la información en el cerebro del sujeto que los resuelve; dicho proceso requiere el ejercicio de la memoria de trabajo así como de la memoria a corto y largo plazo, e implica no sólo la comprensión del problema sino la selección y utilización adecuada de estrategias que le permitirán llegar a la solución.

Polyá por su parte (1982), considera que en el campo de las matemáticas, la resolución de problemas consiste tanto en un proceso de aprendizaje como en un objetivo en si mismo, así como una técnica básica que debe ser desarrollada.

Para otros autores la resolución de problemas podría ser el proceso mediante el cual se llega a la comprensión de una situación incierta inicialmente, para lo cual se requiere tanto la aplicación de conocimientos previos, como de ciertos procedimientos por parte de la persona que resuelve dicha situación (Gagné, 1971; Ashmore y coautores, 1979). Al respecto Novack plantea por su parte, que la resolución de un problema implica además la reorganización de la información almacenada en la estructura cognoscitiva de la persona que lo resuelve, es decir, que hay aprendizaje, modificándola (Novack; 1982, 1988).

Como resultado de todo lo anterior, se han presentado diferentes propuestas de modelos de enseñanza-aprendizaje de las ciencias basados en la resolución de problemas, en cualquiera de sus enfoques. De cualquier forma, los siguientes aspectos se registran como centrales y se considera que deben ser tenidos en cuenta en la resolución de problemas como parte integrante de las estrategias de enseñanza de las ciencias:

- Compresión del área de conocimiento de a cual fue extraído el problema, es decir, la existencia de un dominio de conocimiento.

- El modelo de resolución deberá ayudar al alumno a plantear hipótesis, así como también a diseñar e implementar estrategias o experimentos que le permitan corroborar o improbar dichas hipótesis.

- La comprobación de la solución constituye la fase final del proceso de solución.

- Los problemas seleccionados deberían ser tomados de una situación natural.

\section{SOBRE LA RESOLUCIÓN DE PROBLEMAS COMO CAMPO DE INVESTIGACIÓN Y ACCIÓN}

\section{Enfoques}

En relación con la resolución de problemas en el campo educativo es posible encontrar literatura a partir de 1910; actualmente constituye una línea fértil de investigación en el ámbito mundial, siendo un campo de trabajo de gran actividad y complejidad en los últimos veinte años.

Dicha complejidad guarda relación por una parte, con la diversidad de concepciones de enseñanza-aprendizaje que subyacen a las investigaciones y por otra, con los problemas 
específicos de investigación. Esto a su vez dificulta la interpretación teórica acerca de la resolución de problemas (Cohen, G., 1983) y la construcción de una visión sólida y global sobre el rol que desempeña en la educación en ciencias naturales (Lópes B., Costa N., 1996), determinando la existencia de diferentes enfoques de investigación que bien se podrían ser resumidos en tres:

- Enseñanza para la resolución de problemas en ciencias naturales.

- Enseñanza sobre la resolución de problemas en ciencias naturales.

- Enseñanza de las ciencias naturales, centrada en la resolución de problemas.

Como es de suponer, tanto la investigación como la enseñanza de las ciencias centradas en la resolución de problemas no pueden excluir del todo la utilización de los dos enfoques restantes.

\section{Principales interrogantes}

Como ilustración de la diversidad enunciada, cabe destacar algunos de los interrogantes planteados frecuentemente en la investigación en este campo:

¿Por qué es importante la resolución de problemas? ¿Qué es resolver problemas? (Garrett, R.M., 1988) ¿Qué significa el término problema en el marco de la enseñanza de las ciencias? ¿Existen diferentes tipos de problemas? ¿Qué tipos de problemas se pueden plantear en la enseñanza de las ciencias? (Sigüenza A.F.; Sáez, M: J:, 1990). ¿Problemas aislados o clases de problemas? (Callejo ML., 1990). ¿Por qué resulta tan compleja la enseñanza para resolver problemas? ¿Cómo diseñar una instrucción orientada a aumentar la capacidad para resolver problemas? ¿Cómo evaluar dicha capacidad? ¿Basta que el profesor elabore un amplio listado de problemas para que el estudiante aprenda a solucionar otros? ¿Hasta dónde el problema de enseñar a resolver problemas se convierte en un problema de aprendizaje? El aprendizaje de la resolución de problemas ¿guarda relación con el problema de transferencia de conocimiento a contextos cotidianos? Si se tiene en cuenta que en la enseñanza de conceptos se indaga acerca de las ideas previas, o concepciones, o esquemas alternativos del alumno ¿qué se debería indagar para el caso de la resolución de problemas; ¿Utilizan los alumnos, durante los procesos de resolución, todos sus conocimientos sobre los conceptos y procedimientos involucrados en los problemas que resuelven? ¿Controlan sus procesos de pensamiento eligiendo los enfoques más adecuados y verificando tanto el proceso como la solución final? (Cobo Lozano, 1990) ¿Son iguales todos los procesos de resolución de problemas? ¿Hay diferentes tipos de resolventes? ¿Los problemas son fijos e independientes de su contexto y del resolvente? ¿Qué diferencia un buen resolvente de otro mediocre? (Gil y coautores, 1988) y otros más.

\section{IMPORTANCIA DE DESARROLLAR UNA LÍNEA DE INVESTIGACIÓN RELACIONADA CON LA RESOLUCIÓN DE PROBLEMAS}

En las últimas décadas se ha venido enfatizando la importancia de transformar la enseñanza-aprendizaje de las ciencias naturales en un proceso de "redescubrimiento más que de transmisión de información, leyes, teorías, modelos y hechos, con base en consideraciones tales como el avance acelerado del conocimiento, la relevancia de cierta información actual en el futuro, aspectos ético-filosóficos sobre los procesos de 
construcción de conocimiento científico y otras, las cuales ponen de manifiesto la necesidad de construir currículos que propendan por la superación de la transmisión de una cantidad cada vez más creciente de información, hacia un conjunto de procesos que permitan comprender la actividad científica, qué distingue esta actividad de otras y en general, que resulten de mayor utilidad para la vida cotidiana de cualquier individuo (Garrett, 1988).

Por otra parte, la revisión de literatura relacionada con currículos sobre enseñanza de las ciencias, pone en evidencia una serie de deficiencias que tienen su origen en una formación académica de estudiantes en la cual se prioriza el aprendizaje memorístico de conocimientos aislados, carentes de significado y trascendencia, susceptibles de ser olvidados fácilmente, formación que potencia:

- El desarrollo memorístico con bajo nivel de profundización de los contenidos y procesos.

- Alto grado de desorganización de conceptos aprendidos.

- Escasa probabilidad de generar nuevos conocimientos y procesos a partir de la reorganización de las estructuras y relaciones entre los conceptos de las disciplinas.

- Pasividad y tendencia a aceptar conocimientos y puntos de vista.

- Desarrollo progresivo de esquemas de pensamiento pobres, rígidos y estereotipados que conducen al estancamiento, a la rutina y a una elaboración intelectual superficial o de bajo nivel cognoscitivo (Amestoy de Sánchez, M., 1993).

Adicionaimente y de acuerdo con los planteamientos de Sigüenza y Sáez (1990), en el caso particular de la biología los conocimientos tradicionalmente se han contemplado y transmitido como una colección de hechos, principios, leyes, reglas e interacciones lógicas, práctica que no favorece el acceso del alumno a conocimientos en dicha disciplina, máxime si se tiene en cuenta el avance significativo de los mismos que determina el surgimiento de problemas y preguntas de interés científico, ético y social, hecho este último común a otras disciplinas científicas, que demandan alternativas para su enseñanza.

A esta altura seria posible plantear una hipótesis: a los estudiantes exitosos de ciencias naturales, posiblemente poseedores de cierta estructura y características de pensamiento o de formas de aprendizaje que les permiten un trabajo más adecuado en este campo que a otro tipo de estudiantes, probablemente se les ha propiciado, en ambientes adecuados de aprendizaje y mediante la realización de acciones sistemáticas y deliberadas el desarrollo de pensamiento creativo, proporcionándoles para ello oportunidades basadas en una educación actualizada que permite el desarrollo de habilidades intelectuales y su aplicación a la solución de problemas de la vida diaria y académica, llevando a que tales estudiantes posean un modo de actuar muy cercano al trabajo científico.

En concordancia con ella, se podría proponer que la meta educativa debería obedecer a una actitud en el aula de clase, donde primordialmente se desarrolle de forma conciente y deliberada, el pensamiento creativo de los estudiantes, propiciando su aprendizaje a través de la resolución de problemas y no de una simple acumulación de contenidos. En otras palabras, el aprendizaje de las ciencias naturales debería basarse en el pensar y el hacer, no reducido a una simple transmisión-asimilación de conocimientos, que 
transcienda la sola reflexión filosófica y/o epistemológica y que se halle al nivel de los desarrollos actuales de los mismos. En concordancia con ello, como consecuencia de tales aprendizajes se desarrolla la capacidad de interpretar, valorar y tomar posición frente a hechos o fenómenos de la vida cotidiana que puedan guardar relación tanto con las ciencias naturales como con otras esferas de la actividad humana.

Retomando el planteamiento inicial acerca de la resolución de problemas aplicable a estas últimas, resulta comprensible asumir esta actividad como una alternativa pedagógica que puesta en práctica desde los grados iniciales hasta niveles educativos superiores, viabiliza una formación tendiente a la respuesta adecuada a un entorno cambiante y altamente interactivo tanto en el ámbito social, como científico y tecnológico.

En conclusión, la importancia de adelantar investigación en la línea Resolución de problemas se basa en la posibilidad de transformar el trabajo de aula aproximándolo a la forma de trabajo de los científicos, convirtiendo aquél en una herramienta educativa de gran utilidad, que permita a los educandos integrar sus nuevos conocimientos a los ya existentes, incluso llevando a cabo las reestructuraciones del caso, de tal manera que aplicando las reglas, teorías y leyes conocidas puedan originar nuevas ideas tendientes a la solución de problemas, desarrollando su espíritu científico y su comprensión de la ciencia.

\section{SOBRE LA LÍNEA}

\section{Fundamentación}

Como se mencionara previamente, en el ámbito de la enseñanza de las ciencias desde hace mucho tiempo se acepta que la educación científica no debe basarse solamente en la introducción de conceptos, leyes y teorías, sino que debe acercar además al estudiante al trabajo científico, viabilizando dicho acercamiento mediante la realización de diversas actividades entre las cuales se destacan las de tipo práctico, dado que éstas si se consideran en su sentido más amplio, deberían contribuir de manera importante al logro del objetivo ya planteado (Hodson, 1992).

En este punto conviene recordar que:

- Así como la resolución de problemas constituye un proceso clave en la enseñanza de las ciencias naturales (Dewey, 1975; Garrett, 1988), el uso de problemas es considerado un componente vital de la enseñanza de las ciencias (Sigüenza y Sáez, 1990).

- Los trabajos prácticos de laboratorio son considerados una variedad de problema.

- Usualmente en la literatura se encuentra una división muy marcada entre los denominados problemas de lápiz y papel" y los trabajos prácticos", dentro de los cuales ocupan especial relevancia los trabajos prácticos de laboratorio.

En relación con este último punto, se ha reconocido que con gran frecuencia los profesores de ciencias utilizan después de la explicación de un tema la realización de problemas de aplicación, con el fin de lograr una mejor comprensión de los aspectos teóricos tratados. Sin embargo, el fracaso de los alumnos frente a esta actividad es muy grande y en el mejor de los casos lo que se consigue es la aplicación mecánica de fórmulas $\mathrm{y} / \mathrm{o}$ definiciones estudiadas con anterioridad. 
Adicionalmente, existe entre el profesorado de ciencias una confusión entre lo que se entiende por problema y lo que son los ejercicios de aplicación. Lo cierto es que los profesores consideran equivalentes estos dos aspectos los cuales se tratan de igual manera en los textos, con el consecuente resultado de hacer creer a los alumnos que están resolviendo problemas, cuando en realidad lo que se está planteando son simples ejercicios de aplicación. Esta situación ha generado además en los alumnos, algo que los sicólogos denominan "fijación funcional", in capacitándolos para resolver verdaderos problemas.

En igual forma, una buena cantidad de literatura señala la importancia de los trabajos prácticos de laboratorio en la enseñanza de las ciencias, los tipos de prácticas que deben existir y que existen en determinados libros de texto (Tamir y García Rovira, 1991) qué hay que renovar en los trabajos prácticos (González, 1992) y otros aspectos relativos al tema.

En ese sentido, algunos resultados de investigaciones realizadas en los últimos años señalan deficiencias que se presentan al realizar prácticas de laboratorio (Payá, 1991) El esfuerzo por superar la enseñanza libresca, centrada en contenidos, generó una serie de propuestas renovadoras en donde los trabajos de laboratorio aparecían como las clave del éxito, no obstante dichas estrategias no han resultado tan simples ni fáciles de aplicar y es así como la investigación didáctica ha puesto en evidencia grandes errores de concepción sobre la naturaleza del trabajo científico y sobre la orientación que se ha venido dando a las prácticas de laboratorio.

A este respecto, Hodson (1992) presenta una crítica fundamentada de los resultados obtenidos con la aplicación de currículos innovadores que pretenden encontrar en los trabajos de laboratorio la solución a los problemas de la enseñanza y el aprendizaje de las ciencias e intenta mostrar los inconvenientes del aprendizaje por descubrimiento y lo no adecuados que resultan para proporcionar una imagen correcta del trabajo científico.

Por el momento, en esta línea se concibe el problema diferenciándolo del ejercicio y se acoge a su clasificación en "reales" o "artificiales" y "abiertos" o "cerrados" según los términos ya expuestos. Por otra parte, la línea se fundamenta en una concepción de ciencia según la cual, ésta constituye una actividad humana, una forma de conciencia social, un sistema ordenado de conocimientos que se ha ido estructurando históricamente, cuya veracidad se comprueba y puntualiza constantemente con el devenir de la práctica social y como resultado de la dialéctica del conocimiento, en los nuevos contextos en que se desarrolla.

Las ciencias naturales a su vez, se consideran como las ciencias de la naturaleza, constituidas por el conjunto de disciplinas que la estudian, tomadas integralmente; su objeto de estudio radica entonces en las diferentes especies de materia, en sus formas de movimiento, en su manera de actuar y manifestar-se en la naturaleza, en sus nexos y leyes y en las formas básicas del ser (Rosental, ludin, 1973).

\section{Objeto del trabajo}

Teniendo en cuenta que la experimentación como parte de la solución de determinados problemas de ciencias requiere como etapa previa a su realización el trabajo teórico, igualmente en términos de problemas, esta línea de investigación se ocupa de los denominados en ella "problemas teóricos" y "problemas experimentales", dado que en esencia, la diferencia fundamental entre unos y otros radica en ejecutar en el laboratorio lo 
diseñado y resuelto a nivel teórico, con base en una serie de conocimientos, habilidades, técnicas y destrezas desarrolladas previamente a partir de una serie de ejercicios prácticos, que tienen por objeto familiarizar al estudiante con las tareas del laboratorio y motivarlo hacia abordajes más complejos.

De igual manera, el trabajo teórico se considera como parte esencial de la resolución de problemas experimentales, en concordancia con algunos de los planteamientos registrados en la literatura.

\section{Propósito de la línea}

En concordancia con lo expuesto, en esta línea se tratará de dar respuesta a interrogantes del tipo de los plantados frecuentemente en la investigación en este campo. De manera particular, se espera que el trabajo en esta línea de investigación permita:

- Establecer el significado del término problema en el marco de la enseñanza de las ciencias en Colombia. en los diversos niveles del sistema educativo.

- Caracterizar el tipo de problemas utilizados de manera predominante en diferentes niveles de la educación en ciencias naturales en el país

- Identificar la forma de realización de los trabajos prácticos de laboratorio en la educación en ciencias naturales en el país, en sus diferentes etapas.

- Establecer niveles de logro en cuanto al aprendizaje, según los abordajes identificados del trabajo práctico en el laboratorio

- Proponer modelos de problemas y alternativas de educación en ciencias naturales, centrados en la resolución de problemas

- Participar en el debate acerca de la educación en ciencias naturales con base en la resolución de problemas.

\section{Acerca de la metodología de investigación}

En concordancia con los requerimientos de los proyectos a través de cuya realización se desarrolla la línea, se emplearán diseños metodológicos cualitativos y! o cuantitativos apropiados para cada caso según la naturaleza y etapa de realización de los diferentes proyectos, en concordancia con lo planteado en el documento marco de investigación en el área. Por consiguiente, se prevé la aplicación de metodologías de investigación educativa en las perspectivas empírico-analítica y humanístico-interpretativa.

Por otra parte, debido a que se espera proponer alternativas educativas y modelos de problemas para la educación en ciencias centrados en la resolución de los mismos, se prevé la utilización de modelos investigativos orientados a la práctica educativa, es decir, a la evaluación y el cambio.

\section{Aportes de otros campos del saber}

De igual manera, el trabajo de investigación en la línea se apoya en los desarrollos de otras disciplinas tales como la sicología cognitiva, la epistemología, la neurobiología, la sociología, la antropología y otros, así como también en los planteamientos y resultados 
provenientes de las demás líneas de investigación del Programa de Doctorado. A su vez, constituye fuente de retroalimentación de las mismas.

\section{Alcances y limitaciones de la línea}

Los alcances de esta línea de investigación tienen gran trascendencia, pues en ella confluye el trabajo de otras líneas del Programa de Doctorado: los procesos de razonamiento se analizan a la luz de la resolución de problemas; la evaluación del desarrollo de diversas habilidades intelectuales a través de la educación en ciencias, se realiza teniendo en cuenta la resolución de problemas; las acciones, conocimientos previos, persistencia y utilización de los mismos se trabajan teniendo en cuenta dicha actividad, al igual que los aspectos relacionados con el trabajo en la línea de inteligencia artificial. Por consiguiente, su trascendencia puede convertirse en un momento dado tanto en una fortaleza como en una debilidad.

\section{BIBLIOGRAFÍA}

AMESTOY DE SANCHEZ. MARGARITA. 1993. EI Desarrollo de habilidades de pensamiento y su aplicación a la enseñanza En: Innovación en la educación universitaria en América Latina. OEA. Proyecto Multirregional de Educación Media y Superior PROMESUP. Programa Regional de Desarrollo Educativo PREDE. P. 139249.

ASHMORE. A. D., FRAZER, M.J.; CASEY, R.J. 1979. Problem Solving and Problem Solving Networks in Chemistry. J. Chem. Educ- Vol 56, p 377-379.

CALLEJO, M. L. 1990 En. Marín N. Condiciones Fundamentadas de EnseñanzaAprendizaje para la Resolución de Problemas en Ciencias. Multilit.

COBO LOZANO, 2. 1996. Análisis de las actuaciones de alumnos de $3^{\circ}$ de BUP en la resolución de problemas que comparan áreas de figuras geométricas. Enseñanza de las Ciencias, 14(2), p. 195-207.

COHEÑ, G. 1983. Psicología Cognitiva Alhambra, Madrid.

DEWEY, J. 1910. How We Think D O Heath, Boston.

DEWEY, J. 1975. Experiences in Education New York Collier Books, New York.

FRAZER, M.J, 1982. Solving Chemical Problems, Chemical Society Review, 11(2), p. 171190.

GAGNE, R.N. 1971. Las condiciones del aprendizaje. Aguilar, Madrid.

GARRETT, M.R. 1988, Resolución de problemas y creatividad: implicaciones para el currículo de ciencias, Enseñanza de las Ciencias. 6(3), p. 224-230.

GIL PÉREZ, D.; MARTINEZ TORREGROSA, J.; SENENT PEREZ, F. 1988. El fracaso en la resolución de problemas de física: una investigación orientada por nuevos supuestos. Enseñanza de las Ciencias, 6(2), p. 131-146. 
GONZÁLEZ, M. 1991. ¿Qué hay que renovar en los trabajos prácticos? Enseñanza de las Ciencias, 10(2), p. 206-211.

HODOSN, D. 1992. Redefining and Reorienting Practical Work in School Science. School Science Review, 73(264) p. 65-68.

KEMPA, R.F. 1986, Resolución de problemas de química y estructura cognoscitiva. Enseñanza de las Ciencias, 4(2), p. 99-110.

LOPES, B.; COSTA, N. 1996. Modelo de enseñanza-aprendizaje centrado en la resolución de problemas: Fundamentación, presentación e implicaciones educativas. Enseñanza de las Ciencias, 14(1), 45-61.

NOVACK, J.D. 1982. Teoría y práctica de la educación. Alianza Editorial. Madrid.

1988. Constructivismo humano: un Consenso emergente. Enseñanza de las Ciencias, 6(3), p 2 13-223.

PAYÁ, J. 1991. Los trabajos prácticos en la enseñanza de la física y de la química. Un análisis critico y una propuesta fundamentada. Tesis doctoral. Universidad de Valencia.

PERALES PALACIOS, F.J. 1993. La resolución de problemas: una revisión estructurada. Enseñanza de las Ciencias, 11(2)m p. 170-178.

POLYÁ, G. 1982, Cómo plantear y resolver problemas. Trillas, México. 10ª ed.

ROSENTAL, M.; IUDIN, P. 1973. Diccionario filosófico. Ediciones Universo. Argentina

SIGÜENZA, A.F y SÁEZ, M.N. 1990. Análisis de la resolución de problemas como estrategia de la enseñanza de la biología. Enseñanza de las Ciencias, 8(3), p. 223-230.

SUMMA, 1997. Lo educación y la riqueza de las naciones, pp. 69-77

TAMIR, P.; GARCÍA ROVIRA. M. DEL PILAR 1992 Características de los ejercicios de prácticas de laboratorio incluidos en los libros de texto de ciencias utilizados en Cataluña. Enseñanza de las Ciencias 10(1) p 3-12.

WOODS. D R.: CROWE: CM., HOFFMAN, T W y WRIGTH, J D 1985 Challenges to Teaching Proolem-solving skills. Chem. 13 Bews (Waterloo University) 155, p. 1-12. 